Open Peer Review on Qeios

\title{
Salvage laparoscopic ultra-radical lymph nodal debulking using yasargil clamps for gynecological malignancies. A feasibility analysis
}

\author{
Mariano Catello Di Donna
}

Funding: The author(s) received no specific funding for this work.

Potential competing interests: The author(s) declared that no potential competing interests exist.

\begin{abstract}
The aim of this study was to analyze the feasibility of laparoscopic ultra-radical lymph nodal debulking using Yasargil clamps in gynecological cancer patients with bulky lymph nodes metastases.
\end{abstract}

Objective. The resection of bulky lymph node in gynecological cancer is a challenging surgical procedure. Considering the risk of intraoperative vascular injury and the related poor surgical outcome, a technique to avoid severe complications is needed. Thus, the aim of this study was to analyze the feasibility of laparoscopic ultra-radical lymph nodal debulking using Yasargil clamps in gynecological cancer patients with bulky lymph nodes metastases.

Methods. We retrospectively evaluated a consecutive series of patients with gynecological malignancies who underwent laparoscopic lymph node debulking using Yasargil clamps between September 2010 and April 2020, at three Italian centers. Surgical and post-operative data was analyzed. Results. Forty-three gynecological cancer patients with bulky pelvic and/or aortic lymph nodes metastases undergoing laparoscopic lymph node debulking surgery using Yasargil clamps were included. Median surgicalprocedure time was $300 \mathrm{~min}$ (range 120-550 min), median estimated blood loss $170 \mathrm{~mL}$ (range $0-700 \mathrm{~mL}$ ). Median size of lymph nodes was $50 \mathrm{~mm}$ (range 25-100). R0 resection was achieved in all cases. Four (9.3\%) intraoperative complications occurred. No conversion to an open abdominal approach was required. There were eight post-operative complications classified grade 2 or worse. There were no cases with intra- or postoperative mortality.

Conclusions: In our experience, in carefully selected gynecological cancer patients with bulky lymph node, laparoscopic lymph node debulking using Yasargil clamps is a valid approach to avoid possible severe vascular intraoperative complications. 